BMJ Open

Diabetes

Research

\& Care

\title{
Glycemic excursion minimization in the management of type 2 diabetes: a novel intervention tested in a randomized clinical trial
}

\author{
Daniel J Cox (D) , ${ }^{1}$ Tom Banton, ${ }^{1}$ Matthew Moncrief, ${ }^{1}$ Mark Conaway, ${ }^{2}$ \\ Anne Diamond, ${ }^{1}$ Viola Holmes, ${ }^{3}$ Joyce Green Pastors, ${ }^{3}$ Anne Wolf, ${ }^{3}$ Kun Fang, ${ }^{1}$ \\ Anthony McCall ${ }^{4,5}$
}

\begin{abstract}
To cite: Cox DJ, Banton T, Moncrief M, et al. Glycemic excursion minimization in the management of type 2 diabetes: a novel intervention tested in a randomized clinical trial. BMJ Open Diab Res Care 2020;8:e001795. doi:10.1136/ bmjdrc-2020-001795
\end{abstract}

- Supplemental material is published online only. To view, please visit the journal online (http://dx.doi.org/10.1136/ bmjdrc-2020-001795).

Received 24 July 2020 Revised 13 November 2020 Accepted 21 November 2020

Check for updates

C) Author(s) (or their employer(s)) 2020. Re-use permitted under CC BY-NC. No commercial re-use. See rights and permissions. Published by BMJ.

For numbered affiliations see end of article.

Correspondence to Dr Daniel J Cox; djc4f@hscmail.mcc.virginia. edu

\section{ABSTRACT}

Introduction This study of adults with type 2 diabetes employed a non-inferiority hypothesis to investigate whether an innovative lifestyle focused on minimizing postnutrient blood glucose (BG) excursions (glycemic excursion minimization (GEM)) would be equivalent or superior to conventional weight loss (WL) therapy in regard to reducing $\mathrm{HbA} 1 \mathrm{c}$, and superior to WL when investigating physical, behavioral and psychological secondary outcomes. The impact of BG feedback on GEM efficacy was also investigated.

Research design and methods 178 adults with type 2 diabetes for $\leq 10$ years, $\mathrm{HbA} 1 \mathrm{c} \geq 6.8 \%$, and not using insulin were randomized to WL $(n=40)$ or one of three versions of GEM. Didactic (GEM-D, $n=39$ ) taught participants to choose low-glycemic load foods, reduce sedentary time and increase moderate routine physical activity. GEM-S $(n=51)$ received GEM-D and systematically measured BG before and after meals and physical activity to educate and motivate food and activity choices. GEM-C $(n=48)$ received GEM-D with continuous glucose monitoring feedback. All participants received 6 hours of group training and BG and activity monitors. Before and 3 months after treatment, participants were assessed for HbA1c, lipids, weight, routine physical activity, nutrition, depression, diabetes empowerment and distress. Results GEM versions did not differ in primary or secondary outcomes, so they were combined for analyses. While WL reduced body mass index (BMI) $(p=0.005)$, GEM demonstrated a greater reduction in $\mathrm{HbA1c}(\mathrm{p}=0.005)$, $\mathrm{BM}$ $(p=0.013)$, carbohydrate intake $(p=0.001), B G$ response to a glucose challenge $(p=0.02)$, and cardiovascular risk $(p=0.003)$. Only GEM participants significantly improved diabetes empowerment, diabetes distress, depressive symptoms, steps/day, and active hours and reduced calories/day. Neither intervention had negative side effects. Conclusions GEM is an effective alternative to WL with respect to physical, behavioral and psychosocial outcomes. Trial registration number NCT03196895.

\section{INTRODUCTION}

Weight loss (WL) lifestyle interventions based on caloric restriction are frequently recommended for individuals with type 2 diabetes

\section{Significance of this study}

What is already known about this subject?

- Weight loss therapy is the conventional and effective lifestyle intervention for adults with type 2 diabetes.

What are the new findings?

- An alternative lifestyle intervention, focused on reducing postnutrient glucose excursions through diet and exercise, resulted in a greater improvement in HbA1c and secondary benefits compared with weight loss therapy.

How might these results change the focus of research or clinical practice?

- These findings suggest that clinicians and patients have a new additional lifestyle intervention option that focuses on reducing postnutrient excursions rather than reducing weight.

(T2D). ${ }^{1}$ From a clinician's perspective, this approach may be difficult for some patients to achieve and maintain. Alternative lifestyle approaches for managing T2D could be beneficial. Recently, the American Diabetes Association concluded: 'Reducing overall carbohydrate intake for individuals with diabetes has demonstrated the most evidence for improving glycemia and may be applied in a variety of eating patterns. ${ }^{2}$ This study investigates an alternative T2D management program based on reducing postprandial glucose excursions through reducing carbohydrate ingestion and increasing routine physical activity.

Pharmacological treatment of T2D uses medications having several different modes of action, which provides more tools for treating the disease. Similarly, a variety of useful lifestyle interventions may help improve glucose outcomes. Postprandial glucose often 
Weight Loss

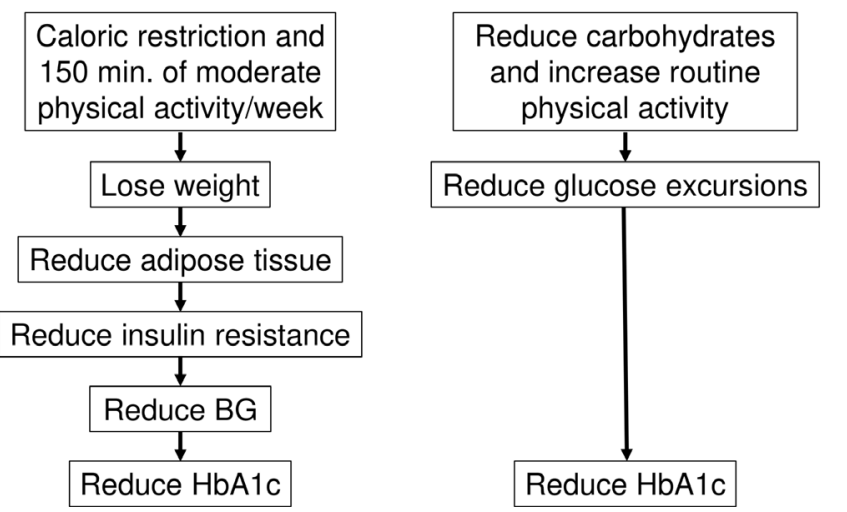

Figure 1 The different mechanisms of action for weight loss $(\mathrm{WL})$ and glycemic excursion minimization (GEM). BG, blood glucose.

represents the highest levels of hyperglycemia reached in many patients with T2D. Glycemic excursion minimization (GEM) focuses on reducing these extremes from meals, snacks and drinks by moderating carbohydrates (which drive hyperglycemia) and increasing routine physical activity after meals (to hasten blood glucose (BG) recovery by using glucose and reducing insulin resistance). ${ }^{3}$

Postprandial glucose excursions are a major contributor to HbAlc and may also independently contribute to diabetes complications. ${ }^{45}$ Our preliminary studies suggested that GEM can reduce both HbA1c and cardiovascular risk. ${ }^{67}$ However, that version of the GEM intervention focused on three specific procedures: (1) replacing high with low Glycemic load foods to diminish BG rises, (2) increasing moderate to vigorous Exercise to hasten BG recovery, and (3) Monitoring BG to learn what impacts BG change. The current GEM intervention focuses on the process of GEM through a variety of procedures not limited to glycemic load, moderate to vigorous exercise, and BG feedback. This study also investigated the contribution of BG monitoring as a feedback procedure, given its cost, inconvenience and past literature indicating minimal efficacy with T2D. ${ }^{8}$

WL and GEM interventions are based on different presumed underlying mechanisms (figure 1). WL interventions reduce HbAlc through a cascade of events where restricting calories (or radically reducing carbohydrates to produce ketones) leads to WL, reduces visceral adipose tissue (a major source of insulin resistance), leads to more efficient insulin action and BG uptake, lowers BG levels, and in turn lowers HbAlc. This chain of processes contrasts with GEM, which directly diminishes BG excursions through reducing carbohydrates and increasing physical activity, subsequently reducing HbA1c. This direct mechanism might produce BG results more quickly, making it evident and encouraging to patients.

Further, reducing glycemic excursions may reduce glucose toxicity and insulin resistance. The term 'glucose toxicity' was originally described as a mixture of insulin resistance due to chronic hyperglycemia and hyperglycemia-induced dysfunction of normal beta cell insulin secretion. ${ }^{9}$ This may occur in a relatively short time (days) although most often it is discussed as a chronic dysfunction contributing to hyperglycemia through multiple mechanisms. ${ }^{10} 11$

Therefore, we hypothesized that GEM would improve the primary outcome variable (HbAlc) and be equivalent or superior (non-inferiority) to an equivalent dose (6 hours of group contact) of conventional WL intervention, but superior to $\mathrm{WL}$ in regard to secondary variables (cardiovascular risk and psychological functioning). Since these interventions are based on different underlying mechanisms, we hypothesized that WL would reduce body mass index (BMI) and calorie ingestion more, while GEM would reduce carbohydrate ingestion and increase physical activity and BG monitoring more. Given the concerns that reducing carbohydrates might result in consuming more fats and worsening lipids, we hypothesized that GEM, compared with WL, would not increase hyperlipidemia. ${ }^{12}$

BG feedback can educate individuals about the effects of their food and activity choices. It may also motivate them to repeat choices that lead to desirable BG levels. It can also serve as a negative feedback loop to activate choices that can correct out-of-control BG levels. ${ }^{13}$ Thus, we hypothesized that increasing the quality and quantity of BG feedback would improve GEM's effectiveness.

\section{MATERIALS AND METHODS \\ Participants}

Two hundred and six adults were recruited through radio and print advertisements and the University of Virginia Hospital patient registry. Twenty-seven failed inclusion/exclusion criteria and one declined to participate (online supplemental table 1). Participants were $30-80$ years old, had T2D for $\leq 10$ years, and had HbA1c $\geq 6.8 \%$. Insulin usage was an exclusion criterion since GEM had the potential to significantly lower BG. Box 1 lists all exclusion criteria. Incentives to participate were free intervention, blood tests, BG meters and supplies (Bayer Contour), activity monitors (Fitbit Charge 2), and $\$ 100$ on completion of the 3-month follow-up assessment. Participants in the different interventions did not differ on any demographic variables (table 1 ).

\section{Procedures}

Assessments

After being thoroughly informed, participants signed a consent form approved by the University of Virginia Institutional Review Board for Health Sciences Research. Next, they participated in a pretreatment assessment including a brief physical, blood tests for HbA1c and lipids, routine consumption of carbohydrates (Carbohydrate Routine Consumption scale (CRC)), psychological questionnaires to assess attitudes towards glucose monitoring, 


\section{Box 1 Exclusion criteria}

Between the ages of 30 and 80 years.

- Type 2 diabetes for 10 years or less.

- $\mathrm{HbA} 1 \mathrm{C} \geq 6.8 \%$.

- Takes insulin. Participants may start insulin during the course of the study if clinically indicated.

Has taken medications that impede weight loss (eg, prednisone) within the last 3 months.

- Currently pregnant or contemplating pregnancy within the next 14 months.

- Has conditions that preclude increasing physical activity (eg, severe neuropathy cardiovascular disease, chronic obstructive pulmonary disease/emphysema, osteoarthritis, stroke, or severe mental disease like manic depressive illness, severe depression, active substance abuse).

- Has conditions that restrict diet, such as severe gastroparesis, ulcers, or food allergies.

- Is undergoing treatment for cancer.

- Has a history of lactic acidosis.

- Has ever had diabetic ketoacidosis.

$\checkmark$ Has marked renal impairment (estimated glomerular filtration rate $<45$; chronic kidney disease stage $3 b$ ).

Takes psychotropic medications that raise blood glucose (eg, atypical antipsychotics).

- Cannot read English.

- Blindness.

Has cognitive impairment.

diabetes empowerment, diabetes distress (Emotional and Regimen subscales), depressive symptoms, and diabetes knowledge as it relates to GEM principles. ${ }^{6}{ }^{14-18}$ Using the Medication Effect Score (MES), a participant's diabetes medications and doses were converted to a common metric, their HbAlc lowering potential, and these were summed across the participant's diabetes medications. ${ }^{19}$
The MES is a way of comparing the wide range of diabetes regimens and doses that participants were using. Participants also completed a mixed meal tolerance test (MMTT). This involved measuring BG following an overnight fast, drinking a $237 \mathrm{~mL}$ Boost Original nutrition drink (41 g carbohydrates, $4 \mathrm{~g}$ fat and $10 \mathrm{~g}$ protein), and measuring BG again $60 \mathrm{~min}$ later. The MMTT was intended to document any reduction to a BG challenge following the different interventions.

The following week, all participants wore a blinded activity monitor to quantify baseline sedentary behavior (hours active) and overall activity (total steps). They were also interviewed by telephone on two workdays and one weekend day for the researcher to complete the Automated Self-Administered 24-hour (ASA24) dietary recall to quantify daily consumption of carbohydrates, fats and proteins. ${ }^{20}$ The ASA24 is a computer-driven interview that documents all nutrients consumed, their volume and preparation method to provide detailed micronutrient analyses of that day's intake. This assessment was repeated 3 months after the conclusion of treatment (follow-up), as shown in figure 2.

\section{Treatment}

Following the pretreatment assessment, blocks of 10 participants were randomized to $\mathrm{WL}$ or one of three versions of GEM by using a minimization method that matched interventions on baseline HbAlc, MES and disease duration. ${ }^{21}$

Participants met in groups of 8-11 people for 6 hours over a 2-month period. GEM participants met for four 90 min sessions, with 1 week between sessions 1 and 2, and 3 weeks between sessions 2-3 and 3-4. This was intended to diminish reliance on group support and encourage autonomy. Because nutritionists wanted more frequent

\begin{tabular}{|c|c|c|c|c|c|c|}
\hline & & WL & GEM-D & GEM-S & GEM-C & $P$ value \\
\hline Started treatment & (n) & 40 & 36 & 50 & 46 & \\
\hline Completed 3-month follow-up & (n) & 36 & 33 & 47 & 43 & \\
\hline Age (years) & (Mean/SD) & $58.3 \pm 10.9$ & $54.7 \pm 11.7$ & $54.8 \pm 11.6$ & $58.2 \pm 11.9$ & 0.301 \\
\hline Education (years) & (Mean/SD) & $15.3 \pm 2.6$ & $16.8 \pm 3.2$ & $15.9 \pm 2.5$ & $15.8 \pm 3.6$ & 0.23 \\
\hline Female & (\%) & 55.6 & 69.7 & 63.8 & 51.2 & 0.353 \\
\hline Black & (\%) & 19.4 & 18.2 & 21.3 & 16.3 & 0.924 \\
\hline White & (\%) & 72.2 & 75.8 & 74.5 & 81.4 & \\
\hline BMI & (Mean/SD) & $34.9 \pm 7.1$ & $34.9 \pm 6.5$ & $35.6 \pm 6.6$ & $34.0 \pm 4.7$ & 0.681 \\
\hline Weight (kg) & (Mean/SD) & $100.1 \pm 20.3$ & $99.1 \pm 20.2$ & $102.2 \pm 21.8$ & $95.9 \pm 16.7$ & 0.512 \\
\hline Years with diabetes & (Mean/SD) & $5.5 \pm 3.0$ & $6.1 \pm 3.1$ & $4.8 \pm 3.2$ & $5.7 \pm 3.2$ & 0.278 \\
\hline $\mathrm{HbA} 1 \mathrm{c}$, pre (mmol/mol) & (Mean/SD) & $67 \pm 11$ & $68 \pm 13$ & $65 \pm 12$ & $71 \pm 18$ & 0.229 \\
\hline $\mathrm{HbA1c}$, pre (\%) & (Mean/SD) & $8.2 \pm 1.0$ & $8.4 \pm 1.2$ & $8.1 \pm 1.1$ & $8.6 \pm 1.6$ & \\
\hline MES, pre & (Mean/SD) & $1.06 \pm 0.74$ & $1.36 \pm 0.82$ & $1.13 \pm 0.80$ & $1.13 \pm 0.81$ & 0.43 \\
\hline MES, post & (Mean/SD) & $1.17 \pm 0.9$ & $1.31 \pm 0.76$ & $1.13 \pm 0.77$ & $1.13 \pm 0.87$ & 0.76 \\
\hline $\begin{array}{l}\text { Hypoglycemia, self-reported incidence of } \\
\text { levels 1-3 (normalized to occurrences/year) }\end{array}$ & (Mean/SD) & $2.0 \pm 6.2$ & $2.6 \pm 6.1$ & $0.9 \pm 3.0$ & $3.6 \pm 9.2$ & 0.26 \\
\hline
\end{tabular}

BMI, body mass index; GEM, glycemic excursion minimization; MES, Medication Effect Score; WL, weight loss. 
Pre-assessment

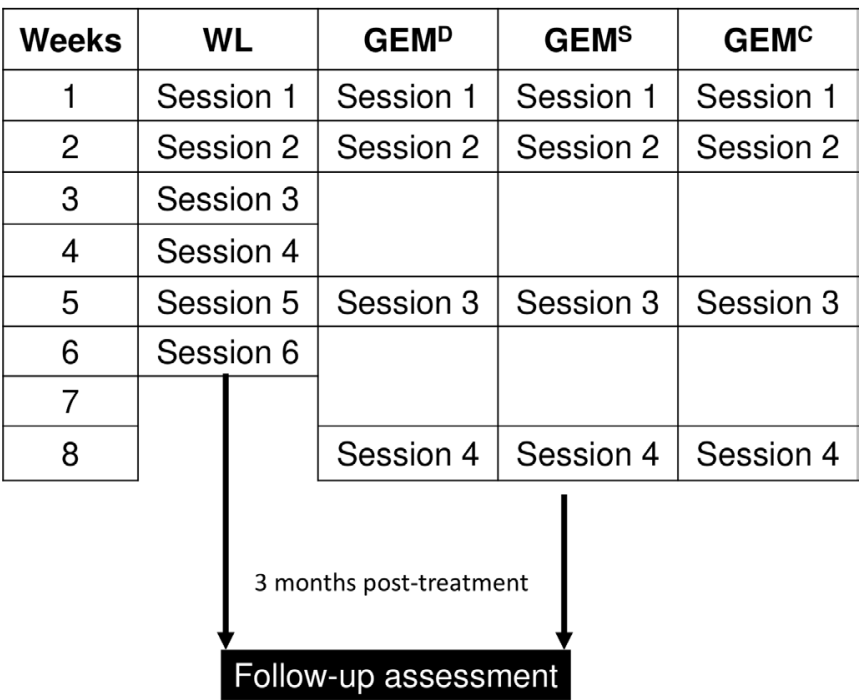

Figure 2 Flow chart illustrating participants' matriculation through the study. GEM, glycemic excursion minimization; WL, weight loss.

contact for WL treatment, WL participants met weekly for six 60 min sessions (figure 2).

All participants received a manual and homework diary (available on request). The manuals for the three GEM versions differed only regarding the inclusion of BG monitoring. GEM-S participants were instructed to measure their BG before and 2 hours after nutrient intake, and before and $30 \mathrm{~min}$ following more than $10 \mathrm{~min}$ of sustained physical activity to learn how these choices impacted their BG. This feedback was also intended to motivate participants to repeat food and activity choices that resulted in desired BG change. GEM-C participants used their continuous glucose monitor (CGM) readouts for similar purposes but noted BG peaks and their duration from such choices. They used the High BG alert to notify them when their BG went above their target and the DexCom Clarity app to review their data. They inserted one 7-day Dexcom G5 sensor at each treatment session, and another 8 weeks after the last treatment session. After the sensors inserted between classes 2-3 and 3-4 expired, participants were encouraged to rely on BG monitoring to note changes in their BG. This was done to promote independence from CGM.

To ensure integrity of the three GEM groups, the diabetes nurse educator (AD) used version-specific instructor's guides and participants used version-specific manuals and diaries. Group sessions comprised participants from the same GEM group. The GEM manual and sessions involved the following four units:

1. Identifying personal motivation to improve metabolic control and educating participants on how their routine choices concerning types and amounts of food and physical activity might affect their BG and their personal goals. In the week following session 1, participants monitored their routine BG-relevant choices in their diary. If assigned to GEM-S or GEM-C, they noted the impact these choices had on their BG.

2. Reducing common high net carbohydrate foods through replacement and substitution. In the 3 weeks following session 2, participants read nutrition labels and recorded choices involving high versus low net carbohydrate foods in their diary. If assigned to GEM-S or GEM-C, they learnt the effect these had on their BG. Participants strove to keep their BG from rising more than $20 \mathrm{mg}(1.665 \mathrm{mmol} / \mathrm{L})$ from premeal levels at 2 hours after ingestion.

3. Increasing mild and moderate physical activity, especially postprandially, to increase direct glucose utilization and enhance insulin sensitivity. In the 3 weeks following session 3, participants used the Fitbit Charge 2 and recorded its feedback in their diary in regard to hours active and total steps. Participants assigned to GEM-S or GEM-C recorded the impact of exercise on their BG.

4. Discussing ways to continue and optimize food and activity choices over a lifetime, to manage relapses, and to thank significant others for their support in making and sustaining their behavior change.

This version of GEM differed from that of an earlier intervention, in that it was group based rather than one on one, did not have a specific fifth unit on BG monitoring, had multiple versions of GEM instead of only GEM-S, and had updated content in the manual. ${ }^{6}$

The WL intervention was led by a registered dietitian/ nutritionist and a certified diabetes educator. It consisted of six, weekly $60 \mathrm{~min}$ group sessions, individualized to accommodate participants' lifestyle and preferences. The goal was to reduce daily calorie intake by $250-500 \mathrm{~g}$ through healthy eating, physical activity, and establishing individualized behavioral goals for eating and activity. The eating plan consisted of healthy carbohydrates (eg, whole grains, fruits, vegetables), lean protein (eg, fish, chicken, legumes), and healthy fats (emphasis on monounsaturated fat). Each week, participants were asked to $\log$ their food intake and physical activity in their diary. The dietitian provided feedback on the logs to help participants achieve their goals. The six-chapter manual was adapted from lessons in the Centers for Disease Control and Prevention curriculum: Prevent $\mathrm{T}^{22}$, and the chapters focused on:

1. eating well and tracking food, with an emphasis on the plate method and basic carbohydrate counting with strategies for tracking intake.

2. getting active and healthy shopping and cooking.

3. burning more calories than are taken in.

4. keeping the heart healthy and managing stress.

5. coping with triggers and taking charge of one's thoughts.

6. eating well away from home and getting support.

WL class activities focused on lessons learnt from the previous week's activities and content from the new chapter. $^{23}$ 
At the beginning of each session, participants in all groups used a standardized questionnaire to report perceived symptoms of all the level 1-3 hypoglycemic experiences they had since the previous session. These were defined as: level 1 (low enough for treatment), level 2 (sufficiently low to indicate serious, clinically important hypoglycemia) and level 3 (associated with severe cognitive impairment requiring external assistance for recovery). ${ }^{18}$

\section{Statistical methods}

The sample size was calculated to provide sufficient power for comparing the primary outcome: change in HbAlc from pre to follow-up, with contrasts used to test specific hypotheses among interventions. Two previous studies of lifestyle intervention in patients with diabetes provided estimates of variation for the calculations. ${ }^{624}$ Allowing for $20 \%$ dropout, a sample of 50 participants per intervention provided $91 \%$ power for the contrast comparing WL and GEM interventions, when the mean HbAlc difference is $0.4 \%$. The sample size also provides $82 \%$ power for comparing the three GEM versions, assuming the difference between the means in the version with the greatest reduction and the version with the least reduction is $0.7 \%$.

Intent-to-treat analyses were performed using SAS V.9.4. A one-way analysis of variance compared changes in HbA1c among the four interventions. Orthogonal contrasts were used to divide the treatment sum of squares, with $3 \mathrm{df}$, to make specific comparisons among the interventions. One contrast $(1 \mathrm{df})$ was used to compare the WL and GEM interventions. The F-test for a second contrast ( $2 \mathrm{df}$ ) was used to compare the three GEM versions regarding changes in HbAlc, followed by pairwise comparisons among GEM versions. $\chi^{2}$ tests were used to compare categorical outcomes among the interventions. Two-tailed probabilities were used to interpret all results. To address possible alpha error from multiple comparisons, the Benjamini-Hochberg procedure was employed. $^{25}$

\section{RESULTS}

\section{Primary outcome variable}

At follow-up, the mean and SD of the HbA1c reduction for GEM-D, GEM-S, and GEM-C was $-0.8 \pm 1.5 \%$, $-1.1 \pm 1.1 \%$, and $-0.9 \pm 1.0 \%, \mathrm{~F}=0.50, \mathrm{p}=0.61 \quad$ (online supplemental table 3 ). Another way of calculating clinical significance is to compare the proportion of participants who lowered their HbA1c below 7.0\%. ${ }^{18}$ After removing four participants whose baseline HbAlc was less than $7.0 \%, 50 \%$ of GEM-D, $50 \%$ of GEM-S, and $34 \%$ of GEM-C achieved this criterion $\left(\chi^{2}=2.52, p=0.284\right)$. Yet another way of contrasting groups is to compare them in regard to the percent responders-those who reduced HbAlc $\geq 0.5 \%$. GEM-D, GEM-S and GEM-C had $64 \%$, $72 \%$, and $67 \%$ who achieved this criterion, respectively $\left(\chi^{2}=0.7, p=0.71\right)$. Furthermore, when the three versions of GEM were compared on secondary variables, they only differed in the frequency of BG monitoring during the third month of follow-up ( $\mathrm{p}=0.001$ ), where GEM-D participants measured BG less frequently (online supplemental table 3). As shown in table 1, the GEM groups did not differ in either occurrence of hypoglycemia during the treatment phase or diabetes medications at baseline and follow-up. Consequently, contrasts were used to compare WL participants to all GEM participants combined.

Table 2 displays pre and follow-up means and SDs for the WL and GEM versions, followed by p levels comparing WL to GEM and pre to follow-up differences for WL and GEM participants. Within each set of contrasts, the error rates are controlled for multiple comparisons using the Benjamini-Hochberg procedure false discovery rate of $0.05{ }^{25}$

Online supplemental table 2 displays pre to follow-up change scores and corresponding $\mathrm{F}$ statistics. Online supplemental figure 1 displays SD differences between the groups.

The mean pre to follow-up reduction of HbAlc was significantly different between GEM and WL: $-0.95 \%$ and $-0.35 \%$, respectively $(\mathrm{F}=8.17, \mathrm{p}=0.005$, table 2$)$. At follow-up, a significantly greater proportion of GEM than WL subjects had HbAlc <7.0\%: $40 \%$ and 23\%, respectively $\left(\chi^{2}=5.255, \mathrm{p}=0.022\right)$. However, GEM did not differ from WL in regard to the percentage of responders; $68 \%$ vs $53 \%$, respectively $\left(\chi^{2}=3.8, p=0.15\right)$.

\section{Secondary outcome variables}

When comparing pre-to-post changes, GEM reduced cardiovascular risk based on the United Kingdom Prospective Diabetes Study Outcomes Model 2 (UKPDS-OM2) ${ }^{26}$ algorithm significantly more than WL $(\mathrm{p}=0.003$, table 2$)$. When comparing pre-to-post changes separately for the GEM and WL groups, only GEM increased empowerment $(\mathrm{p}<0.001)$, decreased diabetes distress on both the Emotional and Regimen subscales $(\mathrm{p}=0.002$ and $\mathrm{p}<0.001$, respectively), and reduced depressive symptoms on the Patient Health Questionnaire-9 ( $\mathrm{p}=0.038)$.

\section{Side effect variables}

Neither WL nor GEM significantly increased consumption of fat or protein or increased low-density lipoprotein, triglycerides or total cholesterol. However, when comparing pre-to-post changes for the GEM and WL groups separately, both WL and GEM significantly increased high-density lipoprotein (HDL) $(\mathrm{p}=0.002$ and $\mathrm{p}<0.001$, respectively; table 2 ). The groups did not differ in their diabetes medication regimens, either at baseline or at follow-up (see tables 1 and 2). Over the course of treatment, no episodes of level 3 hypoglycemia and few episodes of level 1 and 2 hypoglycemia were self-reported, so these were combined for analysis. Extrapolating to an annual event rate, WL and GEM participants reported the equivalent of 2.0 and 2.3 events/year $(\mathrm{t}=-0.264, \mathrm{p}=0.79)$. 


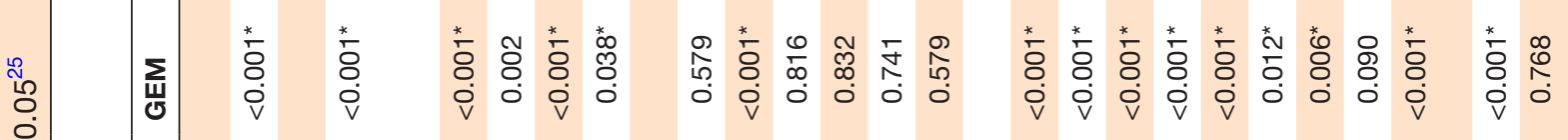
更

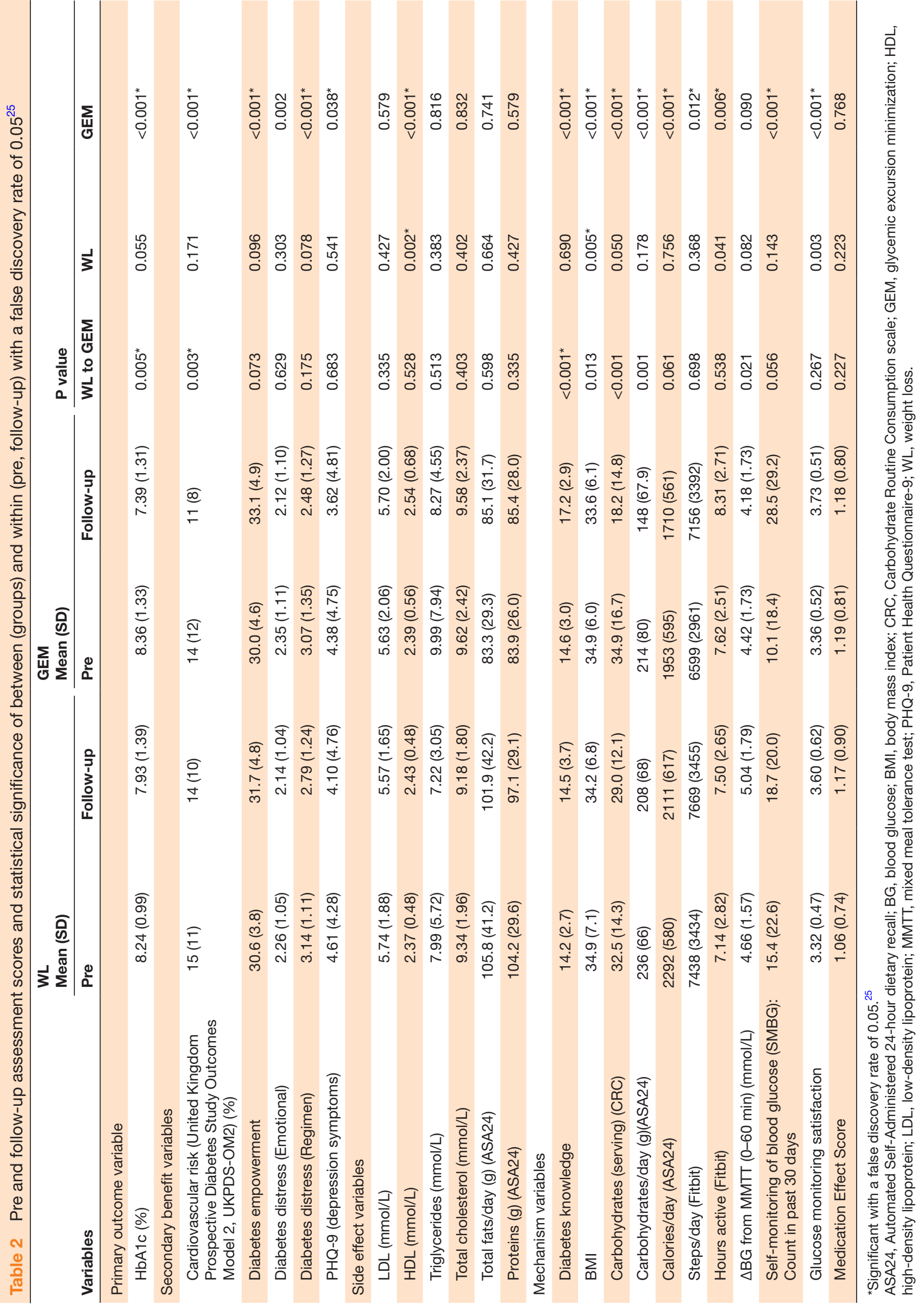




\section{Mechanism variables}

At follow-up, compared with WL, GEM participants demonstrated a greater reduction in BMI $(p=0.013)$ and carbohydrate ingestion on both the CRC and ASA24 (respectively $\mathrm{p}<0.001$ and $\mathrm{p}=0.001$ ), a smaller BG response to Boost $(\mathrm{p}=0.021)$, and an increase in GEM-specific diabetes knowledge $(p<0.001$, table 2$)$. When comparing pre-to-post changes separately for the GEM and WL groups, both WL and GEM participants significantly reduced BMI (respectively $\mathrm{p}=0.005$ and $\mathrm{p}<0.001$ ) and improved Glucose Monitoring Satisfaction Scale scores (respectively $\mathrm{p}=0.003$ and $\mathrm{p}<0.001$ ). Only GEM participants increased their use of self-monitoring of blood glucose $(\mathrm{SMBG}, \mathrm{p}<0.001)$, reduced calories ingested per day on the ASA24 ( $\mathrm{p}<0.001)$, and increased steps/ day $(\mathrm{p}=0.012)$ and hours active $(\mathrm{p}=0.006)$. Groups did not differ in regard to their diabetes medication regimen (MES), both before and after treatment (tables 1 and 2).

\section{DISCUSSION}

This study investigated whether a novel treatment program focused on reducing postnutrient glucose excursions was equal or superior to conventional WL therapy when equating dosage (contact hours). Across primary, secondary and side effect variables, WL was never superior to GEM. This indicates that targeting the reduction of postnutrient BG is a legitimate treatment option for clinicians and patients alike, and may be especially appropriate to consider for those patients who do not need, want, or are unable to achieve and maintain WL.

Both interventions significantly reduced BMI, while increasing HDL and improving attitudes towards BG monitoring. Compared with WL, GEM was significantly better at improving HbAlc, carbohydrate ingestion, BMI, GEM-relevant knowledge, and cardiovascular risk. Further, only GEM significantly improved psychological functioning (greater empowerment, lower diabetes distress, and fewer depressive symptoms), reduced calories, increased physical activity and increased frequency of BG monitoring. Nevertheless, many WL participants did benefit, with 23\% reducing their HbA1c below 7\% and $53 \%$ being classified as responders. The benefits of both interventions and the differences between groups cannot be attributed to change in diabetes medication regimen, as their MES did not change.

The objective of GEM is to empower individuals with the knowledge and skills to make choices that reduce carbohydrate intake and increase routine physical activity. GEM achieved these goals by significantly increasing relevant knowledge, decreasing carbohydrate consumption (as defined by both the CRC questionnaire and the 24-hour dietary recall), and increasing routine physical activity (total steps and hours active) and empowerment. It is important to note that this involved only modest behavior change, with a mean reduction in carbohydrate ingestion of $31 \%$ (follow-up mean consumption of $148 \pm 68$ $\mathrm{g}$ of carbohydrates/day), reduced sedentary behavior and increased steps/day, but no increase in moderate to vigorous activity (Fitbit active minutes). Further, these gains were achieved after only four group sessions combined with educational materials (GEM manual and daily diaries). Additionally, GEM does not involve hunger or restricted calorie intake, and can provide BG feedback within 30-120 min of glycemic-relevant choices.

The empowerment aspect of GEM was hypothesized to reduce depressive symptoms, but this could have also been attributed to the physiological consequences of lowering HbAlc. ${ }^{27}$ An exploratory analysis of GEM participants demonstrated that a reduction in depressive symptoms significantly correlated with reductions in HbA1c ( $r=0.21)$ and diabetes distress (Regimen subscale: $\mathrm{r}=0.43$, Emotional subscale: $\mathrm{r}=0.35$ ), and with an increase in diabetes empowerment $(r=-0.42)$. Stepwise regression indicated that only improvements in diabetes empowerment and the diabetes distress regimen uniquely predicted the reduction in depressive symptoms $\left(R^{2}=0.25\right)$. This suggests that improvement in generic depressive symptoms in patients with T2D may be driven by improvement in diabetes-specific psychological functioning.

There were two unanticipated outcomes. First, while the lack of a significant benefit of BG monitoring among adults with T2D is consistent with some studies, the potential role of BG monitoring to educate, motivate and activate patient choices was anticipated to be beneficial. ${ }^{27}$ The most parsimonious explanation is that the education, exercise, and behavioral feedback from self-monitoring and keeping a diary were powerful enough that glucose monitoring did not add an additional benefit.

A randomized clinical trial methodology may have been inappropriate when comparing different active lifestyle interventions. In this study, participants were randomized to interventions relying on $\mathrm{BG}$ monitoring (GEM-S and GEM-C) or not relying on monitoring (WL and GEM-D). This means that some people who wanted $\mathrm{BG}$ monitoring did not receive it and might have been frustrated, while others who did not want BG monitoring because of needle or blood phobias, apprehension about sensors under their skin, or the technical challenges of BG monitoring received what they did not want. Likewise, it may be that some people who had tried, failed and rejected $\mathrm{WL}$ in the past were randomly assigned to WL. An alternative and possibly more appropriate methodology may be the partially randomized preference design. ${ }^{28}$ This allows patients with strong preferences to select their preferred treatment and randomizes participants with no strong preference to one of the treatment arms. Support for this speculation comes from a small study that the investigators recently conducted where adults were recruited to participate in GEM-C and randomized to that or routine care. ${ }^{29}$ In that study, where everyone who received GEM-C wanted it, the mean HbA1c reduction at the 3-month follow-up was $-1.3 \%$. Further studies will be needed to confirm this.

The second unanticipated outcome was that GEM participants lost more weight than WL participants. 
WL participants reduced their mean intake of carbohydrates, fats and proteins, but these were not significant, and it did not lead to a significant reduction of calories at the 3-month follow-up. In contrast, GEM significantly lowered calorie intake by significantly reducing carbohydrate intake and increasing caloric expenditure through routine physical activity. It may be that focusing on reducing carbohydrates, rather than reducing calories, is a more effective or palatable way to lose weight. ${ }^{2}$

A methodological limitation was providing GEM-D participants with BG meters and unlimited supplies. When these participants asked how often they should measure their BG, they were instructed to follow their primary care physician's recommendations. However, some GEM-D participants used the free SMBG supplies liberally, possibly making them functionally equivalent to GEM-S. Another possible limitation is that our WL intervention was too brief. Despite increasing the number of WL treatment sessions from four to six, we strove to equate doses by giving both interventions 6 hours of group involvement.

Given that GEM and WL were delivered by different clinicians, this could possibly account for differences between the interventions. However, in a program evaluation at follow-up, WL and GEM participants rated how helpful they found listening to the group leader, and hearing from and talking to other participants on a 5-point scale ('Not helpful at all' to 'Extremely helpful'). The respective mean ratings for the WL and GEM interventions on these two questions were 4.4 vs 4.5 ( $p=0.603$ ) and 4.1 vs $4.1 \quad(\mathrm{p}=0.845)$. Further, group attendance was the same for the two interventions, with attendance rates being $80 \%$ and $75 \%$, respectively, for $\mathrm{WL}$ and GEM $(p=0.34)$. These data suggest that the GEM and WL interventions were similar in perceived quality.

Future investigations into the role of BG feedback in GEM should not provide BG monitoring supplies to the equivalent of GEM-D, but should provide regular CGM access to GEM-C participants and employ a partially randomized preference trial design. For greater external validity, it will be important to replicate these findings at other sites and with less restrictive inclusion criteria. It will also be important to identify those individuals who will respond optimally to $\mathrm{WL}$ or to GEM to promote treatment efficacy, reduce patient frustration, and reduce healthcare expenditures.

Despite these limitations, the GEM intervention is a novel and effective treatment for adults with T2D that should be considered as an option for patients who do not want or are unable to lose weight and who are willing to reduce their carbohydrate intake and increase their routine physical activity.

\section{Author affiliations}

${ }^{1}$ Center for Behavioral Medicine Research, University of Virginia School of Medicine, Charlottesville, Virginia, USA

${ }^{2}$ Department of Public Health Sciences, University of Virginia School of Medicine, Charlottesville, Virginia, USA
${ }^{3}$ Virginia Center for Diabetes Prevention and Education, University of Virginia School of Medicine, Charlottesville, Virginia, USA

${ }^{4}$ Department of Medicine, University of Virginia School of Medicine, Charlottesville, Virginia, USA

${ }^{5}$ Division of Nutritional Sciences, Cornell University, Ithaca, New York, USA

Acknowledgements The authors are greatly indebted to their professional advisory board members, Dr Barbara Anderson, Dr Mary DeGroot, and Dr Lawrence Fisher, for their advice on the GEM manual, study design, data interpretation, grant applications, meeting abstracts and manuscripts. They are also indebted to Dr David Repaske for serving as their human subject safety monitor. The authors thank Victoria Ngo and Simone Buckman who managed and assessed the participants, and their research assistants, Emily Klein, Camilla Schanche-Perret Gentil, and Brandon Smith who collected and entered the data.

Contributors DJC oversaw the execution of the project and wrote the manuscript. TB coordinated the project, tested the participants and edited the manuscript. MC oversaw and performed the data analyses. MM managed and analyzed the data. $\mathrm{AD}$ assisted in developing the GEM manual and ran the GEM intervention. $\mathrm{VH}$ and JGP assembled the WL manual. VH, JGP and AW were nutrition consultants and ran the WL intervention. KF performed the literature searches, graphed the findings and assisted in GEM treatment sessions. AM was the endocrinology consultant and edited the manuscript. DJC is the guarantor and is responsible for the contents of this article.

Funding A grant from the NIH/NIDDK (DK108957) funded the study. A grant from Dexcom (IIS-2017-047) provided additional equipment support.

Competing interests None declared.

Patient consent for publication Not required.

Ethics approval This study was performed according to the principles of the Declaration of Helsinki. Ethical approval for this study was obtained from the University of Virginia Institutional Review Board for Health Sciences Research (protocol number 19370).

Provenance and peer review Not commissioned; externally peer reviewed.

Data availability statement Data are available upon reasonable request.

Supplemental material This content has been supplied by the author(s). It has not been vetted by BMJ Publishing Group Limited (BMJ) and may not have been peer-reviewed. Any opinions or recommendations discussed are solely those of the author(s) and are not endorsed by BMJ. BMJ disclaims all liability and responsibility arising from any reliance placed on the content. Where the content includes any translated material, BMJ does not warrant the accuracy and reliability of the translations (including but not limited to local regulations, clinical guidelines, terminology, drug names and drug dosages), and is not responsible for any error and/or omissions arising from translation and adaptation or otherwise.

Open access This is an open access article distributed in accordance with the Creative Commons Attribution Non Commercial (CC BY-NC 4.0) license, which permits others to distribute, remix, adapt, build upon this work non-commercially, and license their derivative works on different terms, provided the original work is properly cited, appropriate credit is given, any changes made indicated, and the use is non-commercial. See: http://creativecommons.org/licenses/by-nc/4.0/.

\section{ORCID iD}

Daniel J Cox http://orcid.org/0000-0002-0910-5306

\section{REFERENCES}

1 Davies MJ, D'Alessio DA, Fradkin J, et al. Management of hyperglycemia in type 2 diabetes, 2018. A consensus report by the American diabetes association (ADA) and the European association for the study of diabetes (EASD). Diabetes Care 2018;41:2669-701.

2 American Diabetes Association (ADA). Standards of medical care in diabetes - 2020. Diabetes Care 2020;43:s1-212.

3 Colberg SR, Zarrabi L, Bennington L, et al. Postprandial walking is better for lowering the glycemic effect of dinner than pre-dinner exercise in type 2 diabetic individuals. J Am Med Dir Assoc 2009;10:394-7.

4 Monnier L, Lapinski $\mathrm{H}$, Colette $\mathrm{C}$. Contributions of fasting and postprandial plasma glucose increments to the overall diurnal hyperglycemia of type 2 diabetic patients: variations with increasing levels of $\mathrm{HbA}(1 \mathrm{c})$. Diabetes Care 2003;26:881-5.

5 Mannucci E, Monami M, Lamanna C, et al. Post-prandial glucose and diabetic complications: systematic review of observational studies. Acta Diabetol 2012;49:307-14. 
6 Cox DJ, Taylor AG, Singh H, et al. Glycemic load, exercise, and monitoring blood glucose (GEM): a paradigm shift in the treatment of type 2 diabetes mellitus. Diabetes Res Clin Pract 2016;111:28-35.

7 Cox DJ, Fang K, McCall AL, et al. Behavioral strategies to lower postprandial glucose in those with type 2 diabetes may also lower risk of coronary heart disease. Diabetes Ther 2019;10:277-81.

8 Young LA, Buse JB, Weaver MA, et al. Glucose self-monitoring in Non-Insulin-Treated patients with type 2 diabetes in primary care settings: a randomized trial. JAMA Intern Med 2017;177:920-9.

9 Rossetti L, Giaccari A, DeFronzo RA. Glucose toxicity. Diabetes Care 1990;13:610-30.

10 Moran A, Zhang HJ, Olson LK, Poitout V, Roberston RP, et al. Differentiation of glucose toxicity from beta cell exhaustion during the evolution of defective insulin gene expression in the pancreatic islet cell line, HIT-T15. J Clin Invest 1997;99:534-9.

11 Robertson R, Zhou H, Zhang T, et al. Chronic oxidative stress as a mechanism for glucose toxicity of the beta cell in type 2 diabetes. Cell Biochem Biophys 2007;48:139-46.

12 Sumithran P, Proietto J. Ketogenic diets for weight loss: a review of their principles, safety and efficacy. Obes Res Clin Pract 2008;2:1-13.

13 Cox DJ, Basu R, McCall A. Continuous glucose monitoring functions as a negative feedback loop for individuals with type 2 diabetes. Ann Diabetes Metab Disord Control 2018;1:121.

14 Polonsky WH, Fisher L, Hessler D, et al. Development of a new measure for assessing glucose monitoring device-related treatment satisfaction and quality of life. Diabetes Technol Ther 2015;17:657-63.

15 Anderson RM, Funnell MM, Fitzgerald JT, et al. The diabetes Empowerment scale: a measure of psychosocial self-efficacy. Diabetes Care 2000;23:739-43.

16 Polonsky WH, Fisher L, Earles J, et al. Assessing psychosocial distress in diabetes: development of the diabetes distress scale. Diabetes Care 2005;28:626-31.

17 Kroenke K, Spitzer RL, Williams JB. The PHQ-9: validity of a brief depression severity measure. J Gen Intern Med 2001;16:606-13.

18 International Hypoglycaemia Study Group. Glucose concentrations of less than $3.0 \mathrm{mmol} / \mathrm{L}$ (54 mg/dL) should be reported in clinical trials: a joint position statement of the American diabetes association and the European association for the study of diabetes. Diabetes Care 2017;40:155-7.
19 Alexopoulos A-S, Yancy WS, Edelman D, et al. Clinical associations of an updated medication effect score for measuring diabetes treatment intensity. Chronic IIIn 2019;17:174239531988409.

20 Subar AF, Kirkpatrick SI, Mittl B, et al. The automated selfadministered 24-hour dietary recall (ASA24): a resource for researchers, clinicians, and educators from the National cancer Institute. J Acad Nutr Diet 2012;112:1134-7.

21 Pocock SJ, Simon R. Sequential treatment assignment with balancing for prognostic factors in the controlled clinical trial. Biometrics 1975;31:103-15.

22 Centers for Disease Control \& Prevention, National Diabetes Prevention Program. Tools and resources: curricula and handouts. Available: https://www.cdc.gov/diabetes/prevention/resources/ curriculum.html?CDC_AA_refVal=https $\% 3 \mathrm{~A} \% 2 \mathrm{~F} \% 2 \mathrm{Fwww}$. cdc.gov\%2Fdiabetes\%2Fprevention\%2Flifestyle-program\% 2Fcurriculum.html [Accessed 30 Apr 2020]

23 Wolf AM, Conaway MR, Crowther JQ, et al. Translating lifestyle intervention to practice in obese patients with type 2 diabetes: improving control with activity and nutrition (ICAN) study. Diabetes Care 2004;27:1570-6.

24 Look AHEAD Research Group, Wing RR, Bolin P, et al. Cardiovascular effects of intensive lifestyle intervention in type 2 diabetes. N Engl J Med 2013;369:145-54.

25 Benjamini Y, Hochberg Y. Controlling the false discovery rate: a practical and powerful approach to multiple testing. $J R$ Stat Soc $B$ 1995;57:289-300.

26 Hayes AJ, Leal J, Gray AM, et al. UKPDS outcomes model 2: a new version of a model to simulate lifetime health outcomes of patients with type 2 diabetes mellitus using data from the 30 year United Kingdom prospective diabetes study: UKPDS 82. Diabetologia 2013;56:1925-33.

27 Geraets AFJ, Köhler S, Muzambi R, et al. The association of hyperglycaemia and insulin resistance with incident depressive symptoms over 4 years of follow-up: The Maastricht Study. Diabetologia 2020;63:2315-28.

28 Bradley-Gilbride J, Bradley C. Partially Randomized Preference Trial Design. In: Salkind NJ, ed. Encyclopedia of research design. USA: Sage, 2010: 1009-15.

29 Cox DJ, Banton T, Moncrief M, et al. Minimizing glucose excursions (GEM) with continuous glucose monitoring in type 2 diabetes: a randomized clinical trial. J Endocr Soc 2020;4:bvaa118. 